\title{
Creciendo en la adversidad. Una revisión del proceso de adaptación al diagnóstico de cáncer y el crecimiento postraumático
}

\section{Growing through adversity. Analysis of the process of adaptation to a diagnosis of cancer and subsequent posttraumatic growth. A review}

\author{
María del Mar Campos-Ríos \\ Universidad de Málaga, España \\ Disponible online 30 de enero de 2013
}

\begin{abstract}
El presente artículo expone un análisis del proceso de adaptación al diagnóstico de cáncer, del cáncer como trauma y del proceso de crecimiento postraumático mediante una revisión de la bibliografía disponible. En primer lugar, se expone el modelo de adaptación a la enfermedad de cáncer de Folkman y Greer (2002) actualizado por Holland (2002); en segundo lugar, se lleva a cabo un análisis del cáncer como posible generador de estrés postraumático para acabar con una revisión del concepto de crecimiento postraumático de Calhoun y Tedeshi (2004), una exposición de las diferencias de opiniones que existen al respecto de este concepto y la sugerencia de futuras líneas de investigación.

Palabras clave: Cáncer; Crecimiento Postraumático; Estrés Postraumático; Adaptación.

The aim of this paper was to examine adjustment to a diagnosis of cancer, cancer as trauma, and the posttraumatic growth process by reviewing the scientific literature. We first present Folkman \& Greer's (2000) adjustment to cancer model updated by Holland (2002) and then review cancer as a possible cause of posttraumatic stress. Finally, we describe Tedeschi \& Calhoun's (2004) Posttraumatic Growth construct. The review is concluded with a sample of different points of view about posttraumatic growth related to cancer, and with some suggestions about future researches.
\end{abstract}

Key words: Cancer; Posttraumatic Growth; Posttraumatic Stress; Adjustment.

Correspondencia: Ma del Mar Campos Ríos. C/ Lince, nº 1, 29190- Málaga. E-mail: mar.campos.rios@gmail.com La autora quiere agradecer a Margarita Ortiz-Tallo su ánimo y colaboración en la elaboración de este trabajo. 
En los últimos años son diagnosticadas de cáncer cada día 10.000.000 de personas en todo el mundo (Maraculla, Ramos y Tabernero, 2009). En España, los últimos datos publicados y avalados por la Sociedad española de Oncología Médica (SEOM) señalan una incidencia de todos los tipos de cáncer, a excepción del cáncer de piel no melanoma, en torno a los 187.573 en 2006, calculando una cifra esperada de más de 200.000 nuevos casos en el año 2020 (Sánchez et al., 2010). Al mismo tiempo, como indica Meseguer (2003), los procesos y reacciones que tengan lugar en cada persona tras afrontar esta experiencia de sufrimiento dependerán de una serie compleja de factores relacionados tanto con la enfermedad específica como con la historia de vida del afectado, incluida su personalidad y sus apoyos. Barroilhet, Forjaz y Garrido (2005) y Spencer, Carver y Price (1998) aseguran que tener cáncer implica experimentar una serie de acontecimientos estresantes interconectados más que un acontecimiento aislado; por lo tanto, en este sentido resulta muy difícil aislar los elementos concretos que pueden generar dicho estrés Así, parece que ser diagnosticado de cáncer, como evento estresante y potencialmente traumatizante, posee unas características especiales (Sumalla, Ochoa y Blanco, 2009) en las que se profundizará más adelante.

El objetivo del presente artículo es exponer un análisis del proceso de adaptación al diagnóstico de cáncer, del cáncer como trauma y del proceso de crecimiento postraumático mediante una revisión de la bibliografía disponible.

\section{Proceso de adaptación a un diagnóstico oncológico}

Las guías del National Cancer Institute (2009) definen la adaptación psicosocial o ajuste al cáncer como un proceso prolongado en el tiempo a través del cual el paciente oncológico trata de conducir el sufrimiento emocional, solventar los problemas concretos relacionados con la enfermedad y ejercer cierto manejo sobre las circunstancias que van surgiendo en torno a la dolencia. Por lo que, conectando con la idea de Barroilhet et al. (2005) y Spencer et al. (1998) anteriormente mencionada, la adaptación a este evento tampoco será un acontecimiento aislado en el tiempo sino que se tratará de una serie continua de respuestas y esfuerzos por parte del enfermo por reajustar su comportamiento ante los continuos cambios internos y externos que padecer cáncer implica.

De este modo, queda reflejada de una forma muy general la noción de que la respuesta ante la enfermedad va a ser diferente en la mayoría de los casos; no obstante, teniendo en cuenta la idiosincrasia de cada individuo, cabe señalar que cuando se habla de estilo de afrontamiento se hace referencia a la estrategia más común, más frecuente y más a largo plazo que tiende a utilizar una persona particular para solventar las diferentes dificultades que encuentra a lo largo de su vida (Barroilhet et al., 2005).

Continuando con esta idea, cabe destacar las aportaciones de Greer, Moorey y Watson (1989) y Watson y Homewood (2008) quienes, a partir de sus investigaciones a través el Mental
Adjustment to Cancer Scale (MAC; Watson et al., 1988), señalan la manifestación de cinco tipos principales de respuesta ante la enfermedad de cáncer, es decir, tendencias en las repuestas de afrontamiento que manifiestan las personas a las que se les comunica un diagnóstico de cáncer; estas son: espíritu de lucha, caracterizado por la aceptación total del diagnóstico, decisión de luchar contra la enfermedad y actitud optimista; desamparo/ desesperanza, definida por la dificultad para pensar en algo que no sea la enfermedad y una actitud totalmente pesimista; preocupación ansiosa, en la que la persona reacciona con una elevada ansiedad, acompañada de ánimo depresivo y búsqueda activa de información que tiende a interpretar de forma pesimista; fatalismo (aceptación estoica), en la que la persona asume el diagnóstico con una actitud derrotista; y finalmente, negación, caracterizada por la negativa a aceptar el diagnóstico o en el caso de aceptarlo, niega o minimiza su seriedad. En la adaptación española de dicho cuestionario (Costa y Gil, 2009) el análisis factorial exploratorio arroja los mismos constructos a excepción de dos nuevos factores en los que quedaría dividido el estilo de afrontamiento espíritu de lucha, estos son, aceptación de la enfermedad y conductas de autocuidado. Sin embargo, estos resultados no se han replicado debido a la ausencia de estudios posteriores al respecto.

En la clasificación original los autores señalaron que la morbilidad psicológica entre mujeres con cáncer de mama se encuentra relacionada con dichas respuestas de afrontamiento, esto es, observaron que la depresión y la ansiedad correlacionan de forma positiva con desamparo/desesperanza, preocupación ansiosa y fatalismo; y negativamente con espíritu de lucha. En este sentido, un reciente estudio con 172 adultos supervivientes de diferentes diagnósticos de cáncer (Schoevers, Kraaij y Garnefski, 2011) indica que las distintas formas de afrontar la experiencia de cáncer generan diferencias en el bienestar psicológico de estas personas, dicho estudio sugiere que la evitación de pensamientos relacionados con la enfermedad se relaciona con un menor nivel de bienestar junto con un menor nivel de autoconfianza, peores relaciones sociales y un menor sentido vital; en definitiva, dicho estilo de afrontamiento basado en la negación potenciaría las experiencias negativas. Por otro lado, las estrategias de afrontamiento relacionadas con una reevaluación positiva y reestructuración de objetivos vitales se relacionan con mayores experiencias positivas, esto es, con un mayor nivel de crecimiento postraumático. Dicho estudio viene a reafirmar otro estudio anterior realizado con 70 mujeres con cáncer de mama en estadios iniciales (Stanton, Danoff-Burg y Huggins, 2002). En España, la bibliografía al respecto es escasa, no obstante un reciente estudio concluye que los estilos de afrontamiento pasivos junto con la evitación emocional predicen una mayor probabilidad de evolución desfavorable en pacientes de cáncer tras dos años de seguimiento (Cardenal, Cerezo, Martínez, Ortiz-Tallo y Blanca, 2011).

Finalmente, las investigaciones clásicas indican que la mayoría de los pacientes se adaptan a la enfermedad de una 
forma que podría considerarse psicológicamente satisfactoria (Morris, Greer y White, 1977). En un estudio llevado a cabo por Simpson, Carlson, Beck y Patten (2002) se observó que el $75 \%$ de las pacientes de cáncer de mama no presentaban síntomas de desajuste psicológico tras completar su tratamiento médico, mientras que el 10\% presentaban síntomas de depresión menor y el 8\% mostraban sintomatología ansiosa. En otro estudio (Grassi, Travado, Moncayo, Sabato y Rossi, 2004) realizado con 277 pacientes el 34\% presentaba ansiedad y el 24,9\% depresión. Recientemente, Turner et al. (2011) y Fischer y Wedel (2012) siguen confirmando estos resultados, indicando que de acuerdo a los criterios del Manual Diagnóstico y Estadístico de los Trastonos Mentales IV (en adelante, DSM-IV) el $30-40 \%$ de los pacientes evaluados mediante una entrevista clínica estructurada presenta algún tipo de trastorno, principalmente depresión, trastorno adaptativo y ansiedad. Finalmente un reciente meta-análisis (Sawyer, Ayers y Field, 2010)_indica que la prevalencia de depresión en pacientes oncológicos es del $14.9 \%$ para el trastorno depresivo mayor y del $19.2 \%$ para los trastornos depresivos menores; por su parte, los trastornos de ansiedad poseen una prevalencia del $10.3 \%$ y el trastorno adaptativo un $19.4 \%$. En cuanto a la prevalencia de estos trastornos en población española, los datos son escasos; Gil et al (2008) señalan que en torno al $24 \%$ de los pacientes de cáncer desarrollan algún tipo de trastorno mental, con prevalencias del $77.3 \%$ para el trastorno adaptativo, un $4.1 \%$ para el trastorno depresivo mayor, $4.4 \%$ para los trastornos de ansiedad y un $2.2 \%$ para el trastorno por estrés postraumático. Este último trastorno se tratará en profundidad más adelante.

Fischer y Wedel (2012) y Newport y Nemeroff (1998) y establecen que entre los factores de riesgo psicosocial de depresión en los pacientes oncológicos se encuentran: pobres habilidades de afrontamiento, escaso apoyo social, presencia de preocupación ansiosa, una edad temprana y una historia previa de problemas psicológicos. Lo anterior se une a que la depresión en pacientes oncológicos suele acompañarse de pensamientos negativos intrusivos relacionados con temas de enfermedad y muerte (Tedeschi y Calhoun, 2004) lo que a su vez parece facilitar el mantenimiento por parte de la persona de estrategias poco adaptativas.

En cuanto al funcionamiento de este proceso de adaptación, actualmente existe un modelo concreto para la enfermedad de cáncer gracias a la adaptación de Folkman y Greer (2000) y la actualización posterior realizada por Holland (2002) del modelo de estrés y afrontamiento de Lazarus y Folkman (1997) que permite visualizar y comprender el camino propuesto por estos autores para afrontar esta enfermedad potencialmente mortal. Según este modelo adaptado y actualizado existen dos procesos básicos: "valoración" y "afrontamiento"; entendiendo por "valoración" la evaluación que cada individuo realiza a partir del significado personal con el que cuenta un evento particular; ello, se entiende, afecta a las emociones así como al "afrontamiento" definido éste como la disposición de los recursos de la persona para resolver ese evento. Desde esta perspectiva se considera que el proceso de estrés comienza en el momento en el que la persona valora la existencia de la enfermedad como algo altamente probable, lo que le lleva a tomar conciencia de un cambio en su realidad y una posible amenaza de sus metas, tal y como las planteaba hasta ahora. En este momento se produce una "valoración primaria" en la que el individuo, influido por sus creencias y valores personales, dará un significado íntimo a esa vivencia pudiendo concebirla como una pérdida, un desafío personal, etc., tras esto, llevará a cabo una "valoración secundaria" en la que realizará un examen de las opciones de afrontamiento en función del grado en el que le es posible cambiar o controlar la situación provocada por el cáncer.

Siguiendo el proceso planteado y de acuerdo con la bibliografía consultada, se entiende el "afrontamiento" como las cogniciones y conductas que la persona lleva a cabo en el intento de adaptarse a la nueva situación relacionada con la enfermedad. De este modo y de acuerdo con el modelo, el paciente puede afrontar el evento mediante tres estrategias generales: centradas en las emociones, centradas en los problemas y centradas en el significado (National Cancer Institute, 2009). Aquellas estrategias centradas en la emoción permiten al enfermo regular el grado de sufrimiento emocional, mientras que aquellas centradas en los problemas facilitan el manejo de las dificultades específicas mediante la modificación de las situaciones que causan malestar y aquellas centradas en el significado permiten buscar un sentido a la enfermedad y al impacto que ella tendrá en su vida tal y como la han conocido hasta ahora. Emplear una estrategia u otra, señalan, dependerá de la intensidad de la respuesta emocional por parte del enfermo, de su habilidad para manejar esta respuesta, de la posibilidad de resolver los problemas que se planteen a raíz de la enfermedad, así como de los cambios que se producen a medida que se desarrolla el proceso entre la persona y su entorno. Por todo lo anterior, es posible rescatar aquí la clasificación elaborada por Watson y Greer en 1989, ya explicada, en la que se establecían cinco tipos básicos de afrontamiento.

A partir de estos eventos internos se plantea un proceso que se retroalimenta en la medida en la que el afrontamiento toma partido en el resultado de la situación y por lo tanto en la valoración posterior que el paciente hará de la nueva situación. Una situación con resultado positivo tenderá a provocar una valoración de ganancia, emociones positivas y la finalización de la estrategia de afrontamiento desplegada; sin embargo ante un resultado insatisfactorio para el paciente el afrontamiento seguirá adelante. De acuerdo con esto, cobra especial importancia la idea manifestada por Folkman y Greer (2000) en la que sugieren que cada persona elabora un "significado global" que se transforma y mantiene a lo largo de toda la vida; dicho significado se forma a partir de sus creencias, valores, metas y autoimagen; este significado global se ve amenazado por un evento trascendental como es el diagnóstico de cáncer a lo que se le otorga un "significado situacional". En este sentido Holland 
(2002) comprende que entonces los esfuerzos de afrontamiento van dirigidos a conciliar ambos tipos de significados a través de las evaluaciones primaria y secundaria hasta llegar a un nuevo equilibrio que permita un afrontamiento eficaz y continuado.

En el siguiente apartado se exponen las características propias del cáncer como suceso traumatizante en relación a dicho "significado situacional" que la persona le otorga en su proceso de afrontamiento.

\section{Cáncer y estrés postraumático}

El cáncer no fue considerado un suceso potencialmente generador de estrés postraumático (en adelante TEPT) de manera oficial hasta la cuarta edición del manual DSM ya que previamente todas aquellas enfermedades médicas potencialmente mortales habían sido intencionalmente excluidas de los manuales diagnósticos como posibles causantes de esta entidad psicológica (Vázquez y Castilla, 2007). A partir de entonces, se admitió que diferentes enfermedades y situaciones vitales difíciles podían potencialmente provocar este trastorno. En este sentido, Kangas, Henry y Bryant (2002) aportan datos sobre la presencia de TEPT en entorno a un $0-5 \%$ en pacientes oncológicos cuando son evaluados mediante entrevista estructurada y en un $5-19 \%$ si se administra un autoinforme; informes más recientes al respecto estiman una prevalencia del 4-6\% (Fischer y Wedel, 2012; Turner-Sack, Menna y Setchell, 2012). En España los escasos datos al respecto indican una prevalencia similar, con un 4.1\% (Gil et al., 2008). Así, parece que el desarrollo de TEPT en estas personas no sólo es posible sino que, como indican algunos autores, posee particularidades relacionadas con las características propias de la enfermedad. Sin embargo, debido a dichas particularidades, la capacidad del cáncer para provocar TEPT no ha estado exenta de discusión. Es por esto que se hace necesario realizar una descripción de las peculiaridades de la enfermedad de cáncer como suceso traumático (Smith, Redd, Peyser y Vogl, 1999; Sumalla et al., 2009):

- En la enfermedad oncológica los estresores se relacionan con una gran variedad de circunstancias por las que el paciente transcurre durante un periodo prolongado de tiempo. Todas las fases del tratamiento implican una gran cantidad de momentos potencialmente adversos que dificultan a la persona poder identificar un único suceso causante de estrés.

- Por otro lado, el cáncer es una enfermedad cuyo origen y desarrollo se produce dentro del individuo por lo que la amenaza no es externa si no que se encuentra dentro de la misma persona, ésto hace que la aparición de sentimientos de culpa y vergüenza sea común en aquellos pacientes que acuden a consulta psico-oncológica.

- Aquí las rumiaciones y constantes recuerdos intrusivos sobre el trauma están más bien dirigidos al futuro. Muchos desarrollan un miedo constante a un futuro incierto desde un presente amenazante.
- A diferencia de un suceso agudo, el diagnóstico de esta enfermedad representa el inicio de un largo periodo de estrés y sufrimiento, una maratón en la que se va a poner a prueba su resistencia una y otra vez a través de, como se ha visto, una amplia cantidad de estresores a lo largo de un considerable periodo de tiempo.

- En sentido algo más halagüeño, sufrir una enfermedad oncológica posee unos matices diferenciadores respecto a un suceso traumático agudo y pasajero. Al poseer carácter interno, esta enfermedad como trauma ofrece la posibilidad de una mayor sensación de control, dados los conocimientos actuales sobre los factores asociados al origen de las enfermedades oncológicas. Según ciertos autores (Sumalla et al., 2009) sería esperable que este elemento característico de la enfermedad como trauma influyera sobre los procesos de desarrollo tanto de TEPT como de crecimiento postraumático. No obstante, más adelante se describirá uno de los modelos que actualmente existen para intentar explicar cómo se produce el crecimiento postraumático a partir de la enfermedad.

Una vez señaladas las características de esta enfermedad como potencialmente generadora de TEPT se da el paso a contemplar la posibilidad de que al mismo tiempo este grave suceso puede poner en marcha los recursos personales de quien la sufre, dando forma a lo que se ha llamado crecimiento postraumático. Por ello, en adelante se describe el concepto y características de este crecimiento en relación a la enfermedad oncológica.

\section{Crecimiento postraumático}

Tedeshi y Calhoun (2004) dos de los autores más relevantes en el ámbito del crecimiento postraumático, lo definen como "el cambio positivo que un individuo experimenta como resultado del proceso de lucha que emprende a partir de la vivencia de un suceso traumático" (p.1). Tomando esta definición y de acuerdo a lo que se ha visto hasta ahora, sería posible considerar que actualmente todo enfermo de cáncer que en su adaptación a la enfermedad indique encontrar elementos positivos en diferentes aspectos de su vida, se puede incluir en la clasificación de crecimiento postraumático como se indica en el estudio llevado a cabo por Schroevers et al. (2011). Es más, y a pesar de lo que más adelante se apuntará al respecto, esto parece ratificarse a partir de diferentes trabajos con diversos tipos de cáncer (Collins, Taylor y Skokan, 1990; Kucukkaya, 2010; Martins da Silva, Moreira y Canavarro, 2011; Tallman, Almaier y García, 2007) en los que se concluye que tras la evaluación los pacientes señalan encontrar beneficios tras el diagnóstico de la enfermedad; esto puede resultar sorprendente en un primer momento ya que resulta difícil imaginar que de una experiencia tan amenazadora para la vida de un individuo sea posible extraer conclusiones positivas; sin embargo, aunque sorprendente, no es extraño ya que cada vez son más numerosos los estudios en los que es posible comprobar cómo muchos de los 
pacientes oncológicos refieren cambios positivos en sus vidas y en ellos mismos a partir de la experiencia con la enfermedad (Mols, Vingerhoets, Coebergh, van de Poll-Franse, 2009; Tallman et al., 2007).

Se ha sugerido que un afrontamiento orientado hacia los problemas podría facilitar este crecimiento; según la literatura incluso en mayor medida que las variables individuales ya mencionadas. En este sentido Bellizzi y Blank (2006) concluyen a partir de sus estudios que aquellas mujeres con cáncer de mama que manifiestan un afrontamiento activo se adaptan mejor a la enfermedad, poseen expectativas más optimistas sobre el cáncer y su tratamiento. Cordova, Cunningham y Carlson (2001) encontraron que aquellas mujeres que hablaban con normalidad de su enfermedad desarrollaban un mayor crecimiento. Esto abre la puerta a la posibilidad de plantear facilitar una mejor adaptación a la enfermedad promoviendo estilos de afrontamiento más activos.

Centrando la vista ahora directamente en la consulta psicooncológica, Coreil, Wilke y Pintado (2004) tras analizar diversos grupos de apoyo concluyen que fomentar el entendimiento de la enfermedad como una oportunidad para enriquecerse y autodescubrirse y no sólo como un enemigo a vencer favorecía encontrar aspectos positivos de la vivencia con la enfermedad. En el mismo sentido, Boyers (2001) concluye que aquellas personas con cáncer que planifican el futuro y recurren al apoyo emocional de los amigos desarrollan mayores niveles de crecimiento. Al mismo tiempo comienzan a aparecer pruebas, aunque aún son escasas, de que aquellas intervenciones cognitivo-conductuales que respeten las reacciones negativas de los pacientes pueden fomentar la búsqueda y la percepción de aspectos positivos en la vivencia de la enfermedad (Antoni et al., 2001). Más allá, Stanton et al. (2002) sugieren que promover la consideración de beneficios en enfermos de cáncer puede provocar efectos saludables.

En relación a los modelos vigentes que tratan de arrojar luz sobre los elementos que participan en los complejos procesos que se dan en el desarrollo del crecimiento postraumático, Tedeshi y Calhoun (2004) sostienen que el cáncer produce una ruptura en el sistema de creencias de la persona que pone en marcha una serie de pensamientos rumiativos de naturaleza automática, estos generan la sintomatología propia del estrés postraumático con el objetivo de reconstruir los esquemas cognitivos alterados por el suceso; con el tiempo esta rumiación automática pasa a ser voluntaria y dirigida a la búsqueda de un sentido de la experiencia de enfermedad. Por lo tanto, parece que es la capacidad que el evento traumático tiene de cambiar los esquemas previos lo que determina que se complete el proceso necesario para que desemboque en un crecimiento personal, más que el evento estresante en sí. Así, en el intento de reestructurar su sistema básico de creencias para que el trauma quede integrado sería posible una transformación. Tras dicha transformación la persona que experimenta un suceso traumático, como una enfermedad que amenaza potencial- mente su integridad física, puede cambiar la forma de entender su realidad interna y externa consagrando lo que puede llamarse, según lo visto hasta el momento, su propio crecimiento postraumático.

En cuanto a las variables individuales asociadas al crecimiento postraumático, parecen existir dos características básicas de personalidad que facilitarían que la persona busque un sentido más positivo a las consecuencias de un evento traumático, estas son, extroversión y apertura a la experiencia (Tedeschi y Calhoun, 2004). A su vez se ha relacionado positivamente con este proceso la presencia de afecto positivo valorado a través de autoestima y sensibilidad al refuerzo (Vázquez y Castilla, 2007).

No obstante, a pesar de que todo lo que se ha visto hasta el momento sugiere la existencia de lo que se conoce como crecimiento postraumático, dentro de este campo existen aún numerosas cuestiones por aclarar; lo que hace que numerosos autores no confíen aún demasiado en la veracidad del mismo, por ejemplo, Zoellner y Maercker (2006) contemplan la posibilidad de concebir lo que se denomina crecimiento postraumático como una ilusión adaptativa a corto plazo que permite al paciente defenderse y compensar el malestar emocional causado por el evento traumático. Aceptando esto como posibilidad se estaría hablando de lo que se ha denominado pseudocrecimiento defensivo. Sin ánimo de caer en esta dicotomía, parece abrirse aquí un interesante campo por explorar en el área del afrontamiento de crisis vitales, en general, y al afrontamiento de la la enfermedad de cáncer en particular. De forma contraria, Vázquez y Castilla (2007) aseguran que los datos actuales indican que la percepción de cambios positivos en pacientes con cáncer es real, no estando asociados con la disposición o los indicadores específicos de evitación concretamente en la situación de los pacientes oncológicos.

Un último punto de interés que se ha encontrado en la revisión realizada indica que el crecimiento postraumático no es, a pesar de que pueda parecerlo, un estado de bienestar; en este sentido se ha sugerido que, de hecho, deben existir circunstancias en las que dicho crecimiento conlleve un incremento del malestar emocional (Tomich y Hegelson, 2004), otros autores como Park, Cohen y Murch (1996) notaron en sus estudios que el hecho de crecer a consecuencia del sufrimiento no conllevaba necesariamente una disminución del estrés provocado por el mismo; más recientemente se ha indicado que las experiencias de cambios positivos y negativos después de un diagnóstico de cáncer son relativamente independientes, es decir, pueden coexistir (Schroevers et al., 2011). No obstante, los datos al respecto parecen inconsistentes, aunque una posible variable mediadora de este proceso podría ser la severidad de la enfermedad según el estudio realizado por Tomich y Hegelson en 2004. Se abre aquí otro interesante campo de investigación para intentar dilucidar las diferentes variables que, teniendo que ver con esta enfermedad concreta, pueden influir en el desarrollo del crecimiento postraumático. 


\section{Conclusión}

En primer lugar, es necesario aclarar que toda la información se ha expuesto en dirección a entender todo el proceso de adaptación a la enfermedad y de crecimiento postraumático en pacientes oncológicos. No obstante, tras la revisión bibliográfica se observó que la gran mayoría de la información al respecto, como con otros muchos aspectos dentro de la PsicoOncología, se dirigía a las mujeres con cáncer de mama; sin embargo, se logró encontrar revisiones en las que se trataban diferentes tipos de enfermedades oncológicas, en muchas de las cuales no se diferenciaban los tipos de cáncer más allá de una somera enumeración de porcentajes de los mismos. Y es por ello que en todo el texto en general y en muchas de las referencias realizadas en particular se habla del cáncer como un campo único. Por otro lado, los datos disponibles con población española son escasos y poco concluyentes. Sin embargo, a pesar de estas limitaciones los datos aquí mostrados pueden resultar estimulantes en dirección a ampliar el conocimiento sobre este campo en nuestro país. Desde aquí parece que cada tipo de cáncer posee sus características propias y provoca también reacciones psicológicas con matices diferentes pero cuando se habla de sufrimiento, se presente dónde se presente, este parece ser universal.

Una vez explicado lo anterior, llama la atención la importancia que parecen tener los estilos de afrontamiento en cuanto al desarrollo o no de crecimiento postraumático; tal y como se ha indicado más arriba, este no parece depender tanto del suceso traumático en sí sino de la capacidad que tenga en una persona particular para cambiar sus esquemas en el intento de recuperar su equilibrio. Aquí podría plantearse la siguiente cuestión: ¿quiere esto decir que para fortalecerse a partir de un suceso traumático es más importante lo que se hace que lo que se es, esto es, cómo uno dirige su comportamiento en los momentos en los que está pasando por circunstancias adversas más que lo que uno es en cuanto a variables de personalidad y experiencia previa? Con esta pregunta se pretende incidir en lo que popularmente se conoce como "sacar fuerzas de flaquezas" y en ese esfuerzo sorprenderse a uno mismo y a partir de ahí también cambiar los esquemas sobre sí y sus capacidades, favoreciendo la autoestima.

Sin embargo, no se debe perder de vista la idea de que el crecimiento postraumático es un proceso complejo que no puede ser reducido únicamente, como indican Tedeschi y Calhoun (2004), a las estrategias de afrontamiento, al cambio en los pensamientos distorsionados, al bienestar psicológico o a cualquiera de los demás factores relacionados, sino que debe contemplarse en su integridad. Al mismo tiempo, no es posible conocer aún las relaciones entre estos factores que componen este proceso ya que hoy día aún se desconoce mucha información sobre el papel de los factores mencionados, lo cual no permite aún predecir o fomentar con propiedad este crecimiento, un meta-análisis llevado a cabo por Prati (2007) indica que aunque la información al respecto es inconsistente parece que las variables asociadas a este crecimiento de forma sistemática son: el optimismo, la espiritualidad, el apoyo social, la búsqueda de apoyo social, el afrontamiento activo, la aceptación y el reencuadre positivo. En este sentido queda todavía mucho por hacer y trabajar. Concretando, una línea interesante en este aspecto sería la construcción de escalas más específicas en relación al crecimiento postraumático relacionado con el cáncer ya que, como se ha podido comprobar, el crecimiento a partir del sufrimiento en esta enfermedad posee características propias que resultaría sumamente interesante conocer con el fin de ahondar en tan estimulante campo de trabajo. En este sentido, Vázquez y Castilla (2007) también indican la necesidad de crear instrumentos de evaluación de crecimiento postraumático más específicos ya que los existentes no incluyen categorías importantes para los pacientes oncológicos como, por ejemplo, la de beneficios relacionados con la salud y los cambios en los hábitos de vida. Ello a su vez indica la posibilidad de que elaborando estas escalas específicas puedan revelarse nuevas variables relacionadas con el crecimiento postraumático más concretamente en poblaciones oncológicas.

Finalmente, es posible abrir una puerta a la esperanza con el conocimiento certero de que no es necesario sufrir un trauma de las características aquí señaladas para poder experimentar este crecimiento. Aunque se ha comprobado que cierto nivel de distrés es necesario para poner los mecanismos del mismo en marcha (Tedeschi y Calhoun, 2004) también la literatura científica revela que es posible experimentar crecimiento sin necesidad de pagar un precio tan alto como el de una enfermedad, ya que numerosas personas refieren experimentar cambios positivos muy relacionados con los que aquí se han tratado sin necesidad de vivenciar ningún evento traumático, esto según McFarland y Alvaro (2000) sería consecuencia de la tendencia en ciertas personas a percibir cambios positivos en sí mismos en general a partir de sus vivencias. No obstante, aunque interesante, este tema excede los objetivos del presente artículo.

En último lugar, indicar que a pesar de que en ocasiones en la Psicología en general y en la Psico-Oncología en particular se tienda, de alguna manera más o menos explícita, a querer aliviar el sufrimiento de los que solicitan ayuda, no se debe perder de vista que, al menos en el sentido de la temática que aquí se ha tratado, ese sufrimiento es natural y parece necesario para que, como se ha visto, impulse a la persona a avanzar en el afrontamiento de su enfermedad, es más, tras todo lo anterior es posible plantear que esos pensamientos rumiativos y dañinos característicos de los pacientes oncológicos y el sufrimiento que presentan podrían ser necesarios para poner su vida en marcha y para comenzar, de estar de acuerdo con su forma de afrontar la vida, el camino hacia su crecimiento personal y poder así salir fortalecidos de una experiencia que en teoría debería debilitar.

Para finalizar, señalar que parece abrirse aquí un camino interesante en la medida en la que este proceso está repleto de paradojas donde se crece personalmente desde la destrucción 
de los propios esquemas, donde el estrés en su máximo nivel parece conducir a un estado de satisfacción, donde la búsqueda de sentido y la voluntad por recuperar el equilibrio permiten a los enfermos de cáncer convertir su enfermedad en una oportunidad que no elegirían si pudieran pero que aprovechan casi sin ser conscientes para, en palabras de muchos de ellos, mejorar su vida y mejorarse a sí mismos. Para acabar, las palabras de alguien que ha experimentado este crecimiento en contacto con el cáncer: “(...) Si pudiera elegir, renunciaría a todo mi crecimiento espiritual e intentaría crecer a mi manera a partir de mis experiencias (...) Pero no puedo elegir." (Viorst, 1986, p. 10).

\section{Referencias}

1. Antoni, M., Lehman, J., Kilbourn, K., Boyers, A., Culver, J., Alferi, S., Yount, S., McGregor, B., Arena, P., Harris, S., Price, A. y Carver, C. (2001). Cognitive-Behavioral Stress management intervention decreases the prevalence of depression and enhances benefit finding among women under treatment for early-stage breast cancer. Health Psychology, 20, 20-32. http://dx.doi.org/10.1037/0278$\underline{6133.20 .1 .20}$

2. Barrioilhet, S., Forjaz, M. y Garrido, E. (2005). Conceptos, teorías y factores psicosociales en la adaptación al cáncer. Actas Españolas de Psiquiatría, 33, 390-397.

3. Boyers, A. (2001). The influence of cognitive-behavioral stress management, optimism, and coping on positive growth in women with breast cancer. (Tesis doctoral). Disponible en Dissertation Abstracts International: Section B: The Sciences and Engineering (61(10- B):5552).

4. Bellizzi, K. M., y Blank, T. O. (2006). Predicting posttraumatic growth in breast cancer survivors. Health Psychology, 25, 47-56. http://dx.doi.org/10.1037/0278-6133.25.1.47

5. Cardenal, V., Cerezo, M.V., Martínez, J., Ortiz-Tallo, M. y Blanca, M.J. (en prensa). Personality, emotions and coping styles: Predictive value for the evolution of cancer patients. Spanish Journal of Psychology.

6. Collins, R., Taylor, S. y Skokan, L. (1990). A better world or a shattered vision? Changes in life perspectives following victimization. Social Cognition, 8, 263-285. http://dx.doi. org/10.1521/soco.1990.8.3.263

7. Cordova. M., Cunningham, L., Carlson, C. y Andrykowski, M. (2001). Posttraumatic growth following breast cancer: a controlled comparison study. Health Psychology, 20, 176185. http://dx.doi.org/10.1037/0278-6133.20.3.176

8. Coreil, J., Wilke, J. y Pintado, I. (2004). Cultural models of illness and recovery in breast cancer support groups. Health Quality Research, 14, 905-923. http://dx.doi. org/10.1177/1049732304266656

9. Costa Requena, G. y Gil, F. (2009). The Mental Adjustment to Cancer Scale: A psychometric analysis in Spanish cancer patients. Psycho-Oncology, 18, 984-991. http://dx.doi. org/10.1002/pon.1466

10. Fischer, D.y Wedel, B. (2012). Anxiety and depression disorders in cancer patients: incidence, diagnosis and therapy. memo - Magazine of European Medical Oncology, 5, 52-54.

11. Folkman, S. (1997). Positive psychological states and coping with severe stress. Social Science \& Medicine, 45, 1207-1221. http://dx.doi.org/10.1016/S02779536(97)00040-3

12. Folkman, S. y Greer, S. (2000). Promoting psychosocial well-being in the face of serious illness: when theory, research and practice inform each other. Psycho-Oncology, 9, 11-9. http://dx.doi.org/10.1002/(SICI)10991611(200001/02)9:1<11::AID-PON424>3.0.CO;2-Z

13. Gil, F., Costa-Requena, G., Pérez, F., Salamero, M., Sánchez, N. y Sirgo, A. (2008). Adaptación psicológica y prevalencia de trastornos mentales en pacientes con cáncer. Medicina Clínica, 130, 90-92.

14. Grassi, L., Travado, L., Moncayo, F. L. G., Sabato, S. y Rossi, E. (2004). Psychosocial morbidity and its correlates in cancer patients of the Mediterranean area: findings from the Southern European Psycho-Oncology Study. Journal of Affective Disorders, 83, 243-248. http://dx.doi. org/10.1016/j.jad.2004.07.004

15. Greer, S., Moorey, S. y Watson, M. (1989). Patient's adjustment to cancer. The mental Adjustment to Cancer (MAC Scale vs. Clinical rating). Journal of Psychosomatic Research, 33, 373-377. http://dx.doi.org/10.1016/00223999(89)90027-5

16. Holland, J.C. (2002). History of psycho-oncology: overcoming attitudinal and conceptual barriers. Psychosomatic Medicine, 64, 206-21.

17. Kangas, M., Henry, J. y Bryant, R. (2002). Posttraumatic stress disorder following cancer. A conceptual and empirical review. Clinical Psychology Review, 22, 499-524. http://dx.doi.org/10.1016/S0272-7358(01)00118-0

18. Kucukkaya, P. G. (2010). An exploratory study of positive life changes in Turkish women diagnosed with breast cancer. European Journal of Oncology Nursing: the Official Journal of European Oncology Nursing Society, 14, 166-73. http://dx.doi.org/10.1016/j.ejon.2009.10.002

19. Macarulla, T., Ramos, F. y Tabernero, J. (2009). Introducción. En T. Macarulla, F. Ramos y J. Tabernero (Ed.s), Comprender el Cáncer (pp. 7-10). Barcelona: Amat.

20. Martins da Silva, S. I., Moreira, H. y Canavarro, M. C. (2011). Growing After Breast Cancer: A Controlled Comparison Study With Healthy Women. Journal of Loss and Trauma, 16, 323-340. http://dx.doi.org/10.1080/15325024 .2011 .572039

21. McFarland, C. y Alvaro, C. (2000). The impact of motivation on temporal comparisons: Coping with traumatic events by perceiving personal growth. Journal of Personality and Social Psychology, 79, 327-343. http://dx.doi. org/10.1037/0022-3514.79.3.327

22. Meseguer, C. (2003). El adulto con cáncer. En M. Die Trill 
(Ed.), Psico-Oncología (pp. 103-114). Madrid: Ades Ediciones.

23. Mols F., Vingerhoets A., Coebergh J. y van de Poll-Franse L. Well-being, posttraumatic growth and benefit finding in long term breast cancer survivors. Psychology and Health, 24, 583-95. http://dx.doi.org/10.1080/08870440701671362

24. Morris, T., Greer, S. y White, P. (1977). Psychological and social adjustment to mastectomy. A two year follow-up study. Cancer, 40, 1381-2387. http://dx.doi. org/10.1002/1097-0142(197711)40:5<2381::AIDCNCR2820400555>3.0.CO;2-B

25. National Cancer Institute. (2009). Adaptación normal, alteración psico-social y trastornos de adaptación. Recuperado de http://www.cancer.gov/espanol/pdq/cuidadosmedicosapoyo/adaptacion/HealthProfessional/ page2

26. Newport D. y Nemeroff C. (1998). Assessment and treatment of depression in the cancer patient. Journal of Psychosomatic Research, 45, 215-37. http://dx.doi. org/10.1016/S0022-3999(98)00011-7

27. Park, C., Cohen, L. y Murch, R. (1996). Assessment and prediction of stress-related growth. Journal of Personality, 64, 71-105. http://dx.doi.org/10.1111/j.1467-6494.1996. tb00815.x

28. Prati, G. (2007). Potential predictors of post-traumatic growth: A meta-analitic study. Psicoterapia Cognitiva $e$ Comportamentale, 13, 13-36.

29. Sánchez, M. J., Payer, T., De Angelis, R., Larrañaga, N., Capocaccia, R. y Martinez, C. (2010). Cancer incidence and mortality in Spain: estimates and projections for the period 1981-2012. Annals of Oncology: Official Journal of the European Society for Medical Oncology / ESMO, 21, 30-36.

30. Schroevers, M. J., Kraaij, V. y Garnefski, N. (2011). Cancer patients' experience of positive and negative changes due to the illness: relationships with psychological well-being, coping, and goal reengagement. Psycho-Oncology, 20, 165172. http://dx.doi.org/10.1002/pon.1718

31. Simpson, J., Carlson, L., Beck C. y Patten, S. (2002). Effect of a brief intervention on social support and psychiatric morbidity in breast cancer patient. Psycho-Oncology, 11, 282-294. http://dx.doi.org/10.1002/pon.565

32. Smith, M. Y., Redd,W. H., Peyser, C. y Vogl, D. (1999). Posttraumatic stress disorder in cancer: A review. Psycho-Oncology, 8, 521-537. http://dx.doi.org/10.1002/(SICI)10991611(199911/12)8:6<521::AID-PON423>3.0.CO;2-X

33. Stanton, A. L., Danoff-Burg, S. y Muggins, M. E. (2002). The first year after breast cancer diagnosis: Hope and coping strategies as predictors of adjustment. PsychoOncology, 11, 93-102. http://dx.doi.org/10.1002/pon.574

34. Spencer, S.M, Carver, C.S. y Price, A.A. (1998). Psychological and social factors in adaptation. En J.C. Holland (Ed.), Psycho-Oncology (pp. 211-222). New York: Oxford University Press.
35. Sumalla, E. C., Ochoa, C. y Blanco, I. (2009). Posttraumatic growth in cancer: reality or illusion? Clinical Psychology Review, 29, 24-33. http://dx.doi.org/10.1016/j. cpr.2008.09.006

36. Sawyer, A., Ayers, S. y Field, A. P. (2010). Posttraumatic growth and adjustment among individuals with cancer or HIV/AIDS: a meta-analysis. Clinical Psychology Review, 30, 436-47. http://dx.doi.org/10.1016/j.cpr.2010.02.004

37. Tallman,B.A.,Altmaier,E.yGarcia,C.(2007).Findingbenefit from cancer. Journal of Counseling Psychology, 54, 481-487. http://dx.doi.org/10.1037/0022-0167.54.4.481

38. Tedeschi, R. G. y Calhoun, L. G. (2004). Posttraumatic growth: Conceptual foundations and empirical evidence. Psychological Inquiry, 15, 1-18. http://dx.doi.org/10.1207/ s15327965pli1501_01

39. Tomich, P. y Hegelson, V. (2004). Is finding good in the bad always good? Benefit finding among women with breast cancer. Health Psychology, 23, 16-23. http://dx.doi. org/10.1037/0278-6133.23.1.16

40. Turner, J., Kelly, B., Clarke, D., Yates, P., Aranda, S., Jolley, D., Chambers, S., et al. (2011). A randomised trial of a psychosocial intervention for cancer patients integrated into routine care: the PROMPT study (promoting optimal outcomes in mood through tailored psychosocial therapies). BMC Cancer, 11, 48. http://dx.doi.org/10.1186/14712407-11-48

41. Turner-Sack, A. M., Menna, R. y Setchell, S. R. (2012). Posttraumatic growth, coping strategies, and psychological distress in adolescent survivors of cancer. Journal of Pediatric Oncology Nursing : Official Journal of the Association of Pediatric Oncology Nurses, 29, 70-9. http://dx.doi. org/10.1177/1043454212439472

42. Vázquez, C. y Castilla C. (2007). Emociones positivas y crecimiento postraumático en el cáncer de mama. Psicooncología, 4, 385-404.

43. Viorst, J. (1986). Necessary losses. New York: Fawcett.

44. Watson, M. y Homewood, J. (2008). Mental Adjustment to Cancer Scale: psychometric properties in a large cancer cohort. Psycho-Oncology, 17, 1146-1151. http://dx.doi. org/10.1002/pon.1345

45. Zoellner, T. y Maercker, A. (2006). Posttraumatic growth in clinical psychology. A critical review and introduction of a two component model. Clinical Psychology Review, 26, 626-653. http://dx.doi.org/10.1016/j.cpr.2006.01.008

Fecha de recepción: 24 de febrero de 2012 Fecha de recepción de la primera versión modificada: 13 de julio de 2012

Fecha de recepción de la segunda versión modificada: 11 de septiembre de 2012 Fecha de aceptación: 21 de septiembre de 2012 